\title{
Patient cumulative radiation exposure-the potential for unintended consequences
}

\author{
Colin Walsh $^{1} \cdot$ Geraldine O'Reilly $^{1} \cdot$ Dara Murphy $^{2}$
}

Received: 9 December 2019 / Revised: 19 February 2020 / Accepted: 10 March 2020 / Published online: 26 March 2020

(C) European Society of Radiology 2020

\begin{abstract}
Several recent publications have sought to highlight the benefits of tracking patient radiation exposure and how it can be used to strengthen the justification and optimization processes to enhance patient safety. In some cases, authors promote the use of dose tracking to keep medical prescribers aware of the patients' cumulative exposure, presumably with a view to influencing the justification process. Inclusion of patient dose history in the justification process can be problematic for reasons that are not always well understood by medical prescribers. Our paper seeks to highlight these issues and attempts to provide some clarification.
\end{abstract}

Key Points

- Care must be taken in how cumulative radiation exposure is interpreted as it pertains to the justification process.

Keywords Radiation protection $\cdot$ Patient safety $\cdot$ Risk $\cdot$ Radiation dosage

\author{
Abbreviations \\ CT Computed tomography \\ DSA Digital subtraction angiography \\ IAEA International Atomic Energy Association \\ LNT Linear no threshold \\ MRI Magnetic resonance imaging
}

In recent times, patient radiation dose tracking systems have become ubiquitous with several publications demonstrating their usefulness in establishing diagnostic reference levels or optimizing radiology examination protocols [1-3].

Additionally, a recent International Atomic Energy Association (IAEA) publication highlighted the usefulness of dose tracking systems in prospectively identifying patient cohorts who experience repeat radiological imaging examina-

Dara Murphy

dara.murphy@olchc.ie

1 Medical Physics \& Clinical Engineering Department, St. James's Hospital, Dublin 8, Ireland

2 Radiology Department, Children's Health Ireland at Crumlin, Dublin 12 , Ireland tions that may give rise to large cumulative radiation exposures. Identification of such cohorts allows for the possibility of changing the patient imaging pathway to reduce the overall cumulative radiation exposure, without affecting the diagnostic outcome [4].

A further benefit of these systems is the generation of patient dose databases that will improve the dosimetry necessary for large-scale epidemiology studies.

However, it is important to be mindful that access to this information can also have unintended consequences if users are not adequately educated with regard to the meaning of cumulative radiation exposures, as understood by current radiation protection models.

As an example, a survey undertaken by the IAEA [5] indicated the following:

- $71.7 \%$ of physicians felt that being aware of previous computed tomography (CT) scans would affect, and they believed improve, referring patients for a CT examination,

- $60.5 \%$ of physicians felt that a system providing them with quick information about patient exposure would be helpful,

- $67.2 \%$ of physicians said they would find it difficult to prescribe a next CT scan to a patient who has already received an effective dose of about $100 \mathrm{mSv}$. 
Although the patient can benefit from a referrer having access to previous imaging when justifying a radiological examination (to avoid unnecessary examinations), the same cannot be said for having access to a patient's cumulative radiation exposure. This metric has no known bearing on a patient's future risk of radiation-induced cancer, as understood by current risk models. If a physician felt obligated to alter the patient's imaging pathway to a less efficacious non-ionizing imaging modality, based on this metric, this could unintentionally negatively impact the patient's medical care.

The use of a patient's cumulative radiation exposure is problematic, primarily due to the misinterpretation of its implication for the justification process. Although there is no explicit statement made in the survey [5] as to why physicians think cumulative dose is important, we suggest the following reasons are likely to be contributory factors:

1. A failure to understand the concept of "sunk cost bias" or

2. A concern that while the sunk cost bias is a wellestablished principle in logic and statistics, it is being applied, in this case, to a radiation risk model which may itself be flawed, thus requiring a clinician to be even more prudential and assume that a patient's exposure to medical ionizing radiation may make them more prone to acquiring a radiation-induced cancer from any future imaging, or

3. A view that, while the evidence for an association between radiation exposure and cancer at low doses is inconclusive, the relationship between radiation exposure and cancer is better substantiated at radiation doses in excess of $100 \mathrm{mSv}$, thus requiring a more prudential approach on behalf of the referring clinician, when a patient accumulates a radiation exposure in excess of this number.

Taking the first point, the IAEA survey appears to indicate that "sunk cost bias", also known as the "gambler's fallacy", is a misunderstanding that persists among the medical profession [5] and may lead to the belief that the risk incurred from previous exposures to ionizing radiation will act to increase the risk of any future exposures. Although discussed in detail elsewhere [6-10], it is likely that many physicians have never heard of the gambler's fallacy as it applies to radiation-induced cancer, and are therefore unlikely to be in a position to take account of it in the first place. This has not been an issue to date, as easy access to patient's cumulative radiation dose history has only recently become possible. However, now that patient dose tracking is becoming ubiquitous, it is essential that physicians gain a clear understanding of the issues around cumulative radiation dose and, in particular, its application to the justification process. This is particularly important for radiologists, who play an important role in ensuring the justification process is not skewed by a misunderstanding of cumulative dose and its relationship to assessing risk and benefit.
In relation to the second point, the relationship between radiation exposure and cancer is based on the linear no threshold (LNT) model which predicts the number of radiationinduced cancers as a function of radiation dose. It is explicit in excluding a lower threshold: according to this model, any exposure to ionizing radiation may cause cancer induction. Although the model is subject to on-going debate, it does not predict that a patient's inherent resistance to radiation detriment is in some way weakened by a single radiological exposure, such that they are left more susceptible to cancer from future radiological examinations.

Of course, it is possible that further advances in radiobiology will find that this model is not correct and that repeat exposure to low levels of ionizing radiation weakens healthy cells, predisposing them to future malignancy. However, it is also possible that the same advances will conclude that the risk of malignancy is less important than currently thought. It may be found that an exposure might produce vulnerability in one patient and promote resistance in another. While research must remain open to all such considerations, radiation protection practice remains driven by prudential models such as the LNT model - a necessity when there is no conclusive evidence to support either side of the argument.

This concern that low-dose exposure may produce a biological vulnerability that increases the chance of a further exposure leading to cancer is not only based on speculation, but on an unquantified speculation. It leads to a vague sense that it might be worse to give an exposure to a patient with a dose history, but does not provide a scientific basis for deciding what to do about this concern. Similarly, in the absence of broader scientific consensus on the issue, it is one's own speculation, and one which conflicts with the current accepted approach to gauging risk (i.e. if we factor in risk from previous exposures, we no longer have a model where risk is linearly proportional to dose for each exposure). Such an approach adds the personal speculation that low-dose radiation causes vulnerabilities which place the patient at increased risk to future exposures, to a theory that is also based on speculation (the LNT is essentially speculative, in the sense that it prudentially assigns a level of risk to exposures where measurable data on risk cannot be reliably obtained) and may encourage an attitude which is overly conservative on radiation risk. This might manifest as reluctance on behalf of a clinician to prescribe a CT scan for a patient that has already had several CT scans.

In relation to the third point, the concern that cumulative radiation exposure for patients who have had a number of scans may exceed $100 \mathrm{mSv}$ has received increased attention recently [4]. This concern centers on there being clearer evidence of a link between radiation exposure and cancer induction at higher doses (i.e. $>100 \mathrm{mSv}$ ). While a dose of over $100 \mathrm{mSv}$ has a greater risk than a dose of less than $100 \mathrm{mSv}$, regardless of whether the dose is received in one or several 
exposures, caution is needed in interpreting the risk at the level of each individual scan.

If we believe a radiation exposure to a patient with a dose history of $100 \mathrm{mSv}$ is in a different risk category to the same exposure where there is no dose history, we end up with the absurd situation where a $1 \mathrm{mSv}$ exposure is believed to be more consequential for a patient with a dose history that exceeds $100 \mathrm{mSv}$, than a $20 \mathrm{mSv}$ exposure for a patient with no dose history. This is not in keeping with the current understanding of radiation-induced cancer, where severity is independent of dose. If it is assumed that low radiation exposures can cause cancer, the effect is as severe (although less probable) for a $1 \mathrm{mSv}$ exposure than for a $20 \mathrm{mSv}$ or a $100 \mathrm{mSv}$ exposure.

With this thinking, there is considerable potential to skew the justification process to the detriment of patient care. It may result in a greater emphasis on risk than is justified for a patient who has had several radiological examinations and as a consequence become an obstacle to access to further diagnostic exams. Conversely, the same thinking would imply a reduced emphasis on risk for patients who have not had previous $x$-ray exposures, which may reduce proper weighting of risk-benefit for these patients.

A further issue that comes up in relation to patients requiring additional radiological exams is whether this changes the case for the exam to be conducted using non-ionizing technologies such as MRI or ultrasound. Two factors must be considered: whether the alternate scan can provide equivalent diagnostic information, and whether the non-ionizing scan can be easily accessed. This latter point adds a further dimension to the risk-benefit equation as it acknowledges that economic factors may also be a consideration in a referral decision.

For example, assume a situation where an MRI scan can answer a particular clinical question, but it is easier to get access to a CT scan. In this case, part of the justification for the CT scan is the lack of availability of MRI scanning. As such, a case could be made that the number of times the patient is unable to access the MRI should inform how we prioritize future access to the MRI scanner. We are not assuming there is a greater risk for subsequent CT scans, only that every CT scan carries a risk. If we do one less $\mathrm{CT}$, then that is one less risk for the patient.

Before completing our discussion, an important caveat to bear in mind is the use of cumulative dose as it applies to deterministic, or tissue, effects. Our discussion has described the limitations of cumulative dose as it applies to assessing the risk of induction of a stochastic effect (e.g. cancer). However, the same logic does not apply to those situations where tissue effects such as skin burns are of concern. For a tissue effect, once a threshold radiation exposure has been exceeded, increasing radiation exposure has the effect of weakening the biological system $[11,12]$. Common outcomes from exceeding these thresholds include skin damage and hair loss. As the radiation dose required to induce a tissue effect is large (typically greater than 2 Gy), they are often not encountered in diagnostic radiology.

Some authors have also reported circumstances where the skin dose from diagnostic imaging modalities such as CT might contribute, in a cumulative sense, and induce tissue effects in skin. Imanishi et al [12] reported hair loss in three patients due to the cumulative radiation dose to skin from a combination of digital subtraction angiography (DSA) and a CT perfusion study. Their study postulated that that the cumulative skin exposure from a combination of CT and DSA, over a short period of time, caused this tissue effect. Where skin doses of the magnitude observed in some interventional radiology/cardiology procedures are expected, knowledge of the additional radiation exposure from subsequent diagnostic imaging modalities, such as CT, can be important. Justification of further diagnostic imaging studies, under these circumstances, may require the prescriber to be cognizant of the cumulative radiation exposure and the potential for a tissue effect to occur.

Finally, the focus of our paper has been on the justification of exams: however, it is also possible that a patient's dose history might prompt increased concern over dose optimization. Care is needed on this point. Diagnostic exams should be optimized whether it is the patient's first or hundredth examination. Dose should not be reduced over a concern about cumulative exposure. Any such adjustment must have, as a primary concern, its effect on image quality [13].

\section{Summary}

We argue that the stochastic risks associated with cumulative dose from previous imaging are in the past and should not influence the justification of further medical diagnostic exposures. Modifying current risk, based on the notional influence of dose history, conflicts with the LNT hypothesis, and has the potential to skew the justification process for ionizing radiation-based exams to the detriment of patient care. The radiation risk from the current scan is the relevant factor in the justification decision.

However, justification decisions are not simply risk-benefit-based: they may also take account of economic factors. Where a patient may prospectively require numerous scans and economic factors influence a referral decision, dose history might be considered when prioritizing access to nonionizing modalities, which can provide equivalent diagnostic information.

Knowledge of cumulative dose is relevant if a potential for tissue injury is a concern. However, this tends to be only applicable to those patients referred for a radiological intervention (e.g. an interventional cardiology study), a small subset of the patient population referred for radiological imaging. 
Funding information The authors state that this work has not received any funding.

\section{Compliance with ethical standards}

Guarantor The scientific guarantor of this publication is Colin Walsh.

Conflict of interest The authors of this manuscript declare no relationships with any companies whose products or services may be related to the subject matter of the article.

Statistics and biometry No complex statistical methods were necessary for this paper.

Informed consent Written informed consent was not required for this study because it does not concern individual patients.

Ethical approval Institutional Review Board approval was not required because it is not a patient study.

\section{Methodology}

- Prospective

\section{References}

1. Boos J, Meineke A, Bethge OT, Antoch G, Kropil P (2016) Dose monitoring in radiology departments: status quo and future perspectives. Rofo 188(5):443-450

2. Fitousi N (2017) Patient dose monitoring systems: a new way of managing patient dose and quality in the radiology department. Phys Med 44:212-221

3. Cournane S, Brunell E, Rowan M (2019) Establishing how patient size and degree of mis-centring affect $\mathrm{CTDI}_{\mathrm{vol}}$ using patient data from a dose tracking system. Br J Radiol. https://doi.org/10.1259/ bjr.20180992
4. International Atomic Energy Agency (2019) Summary of the IAEA technical meeting on radiation exposure of patients from recurrent radiological imaging procedures. Available via https:// wwwiaeaorg/sites/default/files/19/04/rpop-tm_summary_finalpdf Accessed 6 Dec 2019

5. Rehani MM, Berris T (2012) International Atomic Energy Agency study with referring physicians on patient radiation exposure and its tracking: a prospective survey using a web-based questionnaire. BMJ Open 2:e001425

6. Eisenberg JD, Harvey HB, Moore DA, Gazelle GS, Pandharipande PV (2012) Falling prey to the sunk cost bias: a potential harm of patient radiation dose histories. Radiology 263(3):626-628

7. Durand DJ, Dixon RL, Morin RL (2012) Utilization strategies for cumulative dose estimates: a review and rational assessment. J Am Coll Radiol 9:480-485

8. Durand DJ (2011) A rational approach to cumulative effective dose estimates. AJR Am J Roentgenol 197:160-162

9. Walsh C, Murphy D (2014) Should the justification of medical exposures take account of radiation risks from previous examinations? Br J Radiol. https://doi.org/10.1259/bjr.20130682

10. Kofler JM, Jordan DW, Orton CG (2014) Point/counterpoint. Exposure tracking for $\mathrm{x}$-ray imaging is a bad idea. Med Phys. https://doi.org/10.1118/1.4824059

11. Koenig TR, Mettler FA, Wagner LK (2001) Skin injuries from fluoroscopically guided procedures: part 2 , review of 73 cases and recommendations for minimizing dose delivered to patient. AJR Am J Roentgenol 177(1):13-20

12. Imanishi Y, Fukui A, Niimi H et al (2005) Radiation-induced temporary hair loss as a radiation damage only occurring in patients who had combined MDCT and DSA. Eur Radiol. https://doi.org/ 10.1007/s00330-004-2459-1

13. Walsh C, O'Reilly G (2017) An argument against the use of the term dose reduction. International Conference of Radiation protection in Medicine: Achieving Change in Practice. IAEA-CN-255

Publisher's note Springer Nature remains neutral with regard to jurisdictional claims in published maps and institutional affiliations. 